\title{
Parabolic convergence regions of branched continued fractions of the special form
}

\begin{abstract}
Bodnar D.I. ${ }^{1,3}$, Bilanyk I.B. ${ }^{2,3}$
Using the criterion of convergence of branched continued fractions of the special form with positive elements, effective sufficient criteria of convergence for these fractions are established. To study the parabolic regions of convergence, the element regions and value regions technique was used. In particular, half-planes are considered as value regions. A multidimensional analogue of Tron's twin convergence regions for branched continued fractions of the special form is established. The obtained results made it possible to establish the conditions for the convergence of the multidimensional S-fractions with independent variables.

Key words and phrases: branched continued fraction, convergence, continued fraction, rational approximation.
\end{abstract}

${ }^{1}$ West Ukrainian National University, Peremohy Square 3, 46009, Ternopil, Ukraine

${ }^{2}$ Institute for Applied Problems of Mechanics and Mathematics NAS of Ukraine, 3-b Naukova str, 79060, Lviv, Ukraine

${ }^{3}$ Ternopil Volodymyr Hnatiuk National Pedagogical University, 2 Maxyma Kryvonosa Str., Ternopil, Ukraine

E-mail: bodnar4755@ukr.net (Bodnar D.I.), i .bilanyk@ukr . net (Bilanyk I.B.)

\section{Introduction}

Continued fractions are an effective tool for constructing rational approximations of analytic functions. In comparison with power series, they often converge faster, have wider regions of convergence, and have the property of computational stability. The analytic theory of continued fractions is referred in books of O. Perron [23], H. Wall [27], A. Khovanskii [19], W. Jones and W. Thron [18], L. Lorentzen and H. Waadeland [21].

In the 60 s of the twentieth century V.Ya. Skorobogatko proposed to construct rational approximations of the functions of several variables by means of a multidimensional generalization of continued fractions so-called branched continued fractions (BCF). Fundamentals of the analytical theory of general BCF with $N$ branches are given in the books of P.I. Bodnarchuk and V.Ya. Skorobogatko [12], V.Ya. Skorobogatko and D.I. Bodnar [26], D.I. Bodnar [7].

The problem of constructing the corresponding BCF for multiple power series caused the appearance of two-dimensional continued fractions (Kh.Y. Kuchminska [20], M. O'Donohoe and J. Murphy [22], A. Cuyt and B. Verdonk [13], W. Siemashko [25], O.M. Sus [4]) and BCF with independent variables (D.I. Bodnar [8], O.E. Baran [5], T.M. Antonova [2], and R.I. Dmytryshyn $[3,9,10,14])$. Some examples of functions extensions into BCF with independent variables are provided by R.I. Dmytryshyn (see, for example, [16]).

The problem of establishing regions of convergence of BCF with independent variables is one of the most important. Since many convergence criteria of continued fractions are formulated as convergence regions, it is also quite natural to construct such regions, in particular, 
parabolic regions of convergence, for BCF with independent variables. Such regions of convergence were studied in the works $[1,3,5,7,8,11,14,15]$.

A BCF with independent variables at fixed values of variables is called a BCF of the special form. This fraction is written as follows

$$
b_{0}+D_{k=1}^{\infty} \sum_{i_{k}=1}^{i_{k-1}} \frac{a_{i(k)}}{b_{i(k)}}=b_{0}+\sum_{i_{1}=1}^{N} \frac{a_{i(1)}}{b_{i(1)}+\sum_{i_{2}=1}^{i_{1}} \frac{a_{i(2)}}{b_{i(2)}+\sum_{i_{3}=1}^{i_{2}} \frac{a_{i(3)}}{b_{i(3)}+\ddots \ddots}}},
$$

where $b_{0}, a_{i(k)}, b_{i(k)} \in \mathbb{C}, i(k) \in \mathcal{I}$,

$$
\mathcal{I}=\left\{i(k)=\left(i_{1}, i_{2}, \ldots, i_{k}\right): 1 \leq i_{k} \leq i_{k-1} \leq \cdots \leq i_{0} ; k \geq 1 ; i_{0}=N\right\}
$$

We say that the BCF (1) converges if there exists a finite limit of the sequence of its approximants

$$
f_{n}=b_{0}+D_{k=1}^{n} \sum_{i_{k}=1}^{i_{k-1}} \frac{a_{i(k)}}{b_{i(k)}}, \quad n=1,2, \ldots
$$

In this paper, we investigate parabolic regions of convergence and consider some application for them.

\section{Sufficient criteria of convergence of BCF of the special form with positive elements}

The question of the convergence of a BCF of the special form with positive elements is completely solved by the following theorem established in [6], it is the multidimensional generalization of Seidel's criterion (see, for example, [26, p. 14]).

Theorem 1. The BCF of the special form

$$
b_{0}+D_{k=1}^{\infty} \sum_{i_{k}=1}^{i_{k-1}} \frac{1}{b_{i(k)}}
$$

where $b_{0}, b_{i(k)}>0, i(k) \in \mathcal{I}$, converges if and only if the following series diverge

$$
\sum_{p=1}^{\infty} b_{m[p]}^{(m-1)}, \quad m=\overline{1, N}, \quad \sum_{p=1}^{\infty} b_{i(n), m[p]}^{(m-1)}, \quad i(n) \in \mathcal{I}^{(m+1)}, m=\overline{1, N-1},
$$

the elements of which are defined by the relations

$$
\begin{gathered}
b_{i(k)}^{(0)}=b_{i(k)}, \quad i(k) \in \mathcal{I}, \\
b_{m[p]}^{(m-1)}=b_{m[p]}^{(m-2)}+D_{s=1}^{\infty} \frac{1}{b_{m[p], \underline{m-1}[s]}^{(m-2)}}, \quad m=\overline{2, N}, \\
b_{i(n), m[p]}^{(m-1)}=b_{i(n), m[p]}^{(m-2)}+D_{s=1}^{\infty} \frac{1}{b_{i(n), m[p], \underline{m-1}[s]}^{(m-2)}}, \quad i(n) \in \mathcal{I}^{(m+1)}, m=\overline{2, N-1,}
\end{gathered}
$$


where

$$
\begin{gathered}
\mathcal{I}^{(m+1)}=\left\{i(k)=\left(i_{1}, i_{2}, \ldots, i_{k}\right): m+1 \leq i_{k} \leq i_{k-1} \leq \cdots \leq i_{0} ; k \geq 1 ; i_{0}=N\right\}, \\
m[p]=\underbrace{m, m, \ldots, m}_{p} ; p=1,2, \ldots ; \underline{m-1}[s]=\underbrace{m-1, m-1, \ldots, m-1}_{s} ; s=1,2, \ldots .
\end{gathered}
$$

The conditions of this theorem are formulated rather difficult for practical verification, so it is expedient to establish an effective sufficient condition of convergence of the BCF (2).

Theorem 2. The BCF (2) is convergent if the following series are divergent

$$
\sum_{p=1}^{\infty} b_{m[p]}, \quad m=\overline{1, N}, \quad \sum_{p=1}^{\infty} b_{i(n), m[p]}, \quad i(n) \in \mathcal{I}^{(m+1)}, \quad m=\overline{1, N-1} .
$$

Proof. For proving it is enough to show that from the divergence of the series (5) it follows the divergence of the set of series (3) for each $m=\overline{1, N}$.

Let $m=1$. For any $i(n) \in \mathcal{I}^{(2)}$, the series

$$
\sum_{p=1}^{\infty} b_{1[p]}^{(0)} \quad \text { and } \quad \sum_{p=1}^{\infty} b_{i(n), 1[p]}^{(0)}
$$

are divergent by the conditions (5) since $b_{1[p]}^{(0)}=b_{1[p]}, b_{i(n), 1[p]}^{(0)}=b_{i(n), 1[p]}$ for all $p \geq 1$ and $i(n) \in \mathcal{I}$. This fact and the Seidel theorem [26, p.14] imply that the continued fractions

$$
b_{i(n)}^{(1)}=b_{i(n)}^{(0)}+D_{s=1}^{\infty} \frac{1}{b_{i(n), 1[s]}^{(0)}}, \quad i(n) \in \mathcal{I}^{(2)} \text {, }
$$

are convergent.

Let $m=2$. For any $i(n) \in \mathcal{I}^{(3)}$, from the divergense of the series (5), where $m=2$, it follows divergence of the series

$$
\sum_{p=1}^{\infty} b_{2[p]}^{(1)} \quad \text { and } \quad \sum_{p=1}^{\infty} b_{i(n), 2[p]}^{(1)}
$$

since $b_{2[p]}^{(1)}>b_{2[p]}^{(0)}=b_{2[p]}, b_{i(n), 2[p]}^{(1)}>b_{i(n), 2[p]}^{(0)}=b_{i(n), 2[p]}$, where $p \geq 1, i(n) \in \mathcal{I}^{(3)}$.

Therefore, for any $i(n) \in \mathcal{I}^{(3)}$, the continued fractions

$$
b_{i(n)}^{(2)}=b_{i(n)}^{(1)}+D_{s=1}^{\infty} \frac{1}{b_{i(n), 2[s]}^{(1)}}
$$

are convergent according to the Seidel theorem.

By analogy for $m=3,4, \ldots, N-1$, we obtain the divergence of the series

$$
\sum_{p=1}^{\infty} b_{m[p]}^{(m-1)} \text { and } \sum_{p=1}^{\infty} b_{i(n), m[p]}^{(m-1)}
$$

for all $i(n) \in \mathcal{I}^{(m+1)}$, and the convergence of fractions, which are determined according to (4) and take values $b_{r[q]}^{(r-1)}, b_{i(n), r[q]}^{(r-1)}, i(n) \in \mathcal{I}^{(r+1)}, r=4,5, \ldots, N-1$, and fractions $b_{N[q]}^{(N-1)}, q \geq 1$. 
Finally, let $m=N$. In this case, we have the divergence of the series

$$
\sum_{p=1}^{\infty} b_{N[p]}
$$

Taking into account the relations (4), we obtain

$$
b_{N[p]}^{(N-1)}>b_{N[p]}^{(N-2)}>\cdots>b_{N[p]}^{(1)}>b_{N[p]}^{(0)}=b_{N[p]}, \quad p=1,2, \ldots .
$$

Therefore, the series (3) are divergent, and the BCF (2) converges via the sufficiency of Theorem 1.

Let us prove criteria of convergence of a BCF of the special form with arbitrary positive elements.

Theorem 3. The BCF with positive elements

$$
b_{0}+\prod_{k=1}^{\infty} \sum_{i_{k}=1}^{i_{k-1}} \frac{a_{i(k)}}{b_{i(k)}}
$$

converges if the following series diverge

$$
\begin{gathered}
\sum_{p=1}^{\infty} \sqrt{\frac{b_{m[p]} b_{m[p+1]}}{a_{m[p+1]}}}, \quad m=\overline{1, N}, \\
\sum_{p=1}^{\infty} \sqrt{\frac{b_{i(n), m[p]} b_{i(n), m[p+1]}}{a_{i(n), m[p+1]}}}, \quad i(n) \in \mathcal{I}^{(m+1)}, m=\overline{1, N-1} .
\end{gathered}
$$

Proof. We consider the equivalent BCF [7, p. 29] (see also [21]), the approximants of which coincide with the approximants of the BCF (6),

$$
b_{0}+D_{k=1}^{\infty} \sum_{i_{k}=1}^{i_{k-1}} \frac{1}{d_{i(k)}}
$$

where

$$
d_{i(k)}=b_{i(k)} \prod_{p=1}^{k}\left(a_{i(p)}\right)^{(-1)^{k+p-1}}, \quad i(k) \in \mathcal{I} .
$$

According to Theorem 2 the BCF (7) converges if the following series diverge

$$
\sum_{p=1}^{\infty} d_{m[p]}, \quad m=\overline{1, N} ; \quad \sum_{p=1}^{\infty} d_{i(n), m[p]}, \quad i(n) \in \mathcal{I}^{(m+1)}, \quad m=\overline{1, N-1} .
$$

Now we consider partial sums of these series. For each $r \geq 2$ and $m=\overline{1, N}$ we have

$$
\begin{aligned}
\sum_{p=1}^{r} d_{m[p]} & =\frac{1}{2}\left(d_{m[1]}+d_{m[r]}\right)+\sum_{p=1}^{r-1} \frac{1}{2}\left(d_{m[p]}+d_{m[p+1]}\right) \\
& \geq \frac{1}{2}\left(d_{m[1]}+d_{m[r]}\right)+\sum_{p=1}^{r-1} \sqrt{d_{m[p]} d_{m[p+1]}} \\
& >\sum_{p=1}^{r-1} \sqrt{b_{m[p]} \prod_{s=1}^{p}\left(a_{m[s]}\right)^{(-1)^{p+s-1}} b_{m[p+1]} \prod_{s=1}^{p+1}\left(a_{m[s]}\right)^{(-1)^{p+s}}}=\sum_{p=1}^{r-1} \sqrt{\frac{b_{m[p]} b_{m[p+1]}}{a_{m[p+1]}}} .
\end{aligned}
$$


Analogously, for each $r \geq 2, m=\overline{1, N}$ and $i(n) \in \mathcal{I}^{(m+1)}$ we obtain

$$
\begin{aligned}
\sum_{p=1}^{r} d_{i(n), m[p]} & >\sum_{p=1}^{r-1} \sqrt{b_{i(n), m[p]} \prod_{s=1}^{n}\left(a_{i(s)}\right)^{(-1)^{n+p+s-1}} \prod_{s=1}^{p}\left(a_{i(n), m[s]}\right)^{(-1)^{n+p+s-1}}} \\
\times & \sqrt{b_{i(n), m[p+1]} \prod_{s=1}^{n}\left(a_{i(s)}\right)^{(-1)^{n+p+s}} \prod_{s=1}^{p+1}\left(a_{i(n), m[s]}\right)^{(-1)^{n+p+s}}}=\sum_{p=1}^{r-1} \sqrt{\frac{b_{i(n), m[p]} b_{i(n), m[p+1]}}{a_{i(n), m[p+1]}}} .
\end{aligned}
$$

Then, taking into account the divergence of the last series and Theorem 2, we obtain convergence of the BCF (7). Therefore, the equivalent to it the BCF (6) converges as well.

Note that this theorem is multidimensional generalization of Pringsheim's theorem (see, for example, [21, p. 118]).

\section{Parabolic regions of convergence}

In proving parabolic theorems, the elements and value regions technique is significantly used [7, pp. 111-116] (see also [17]).

The sequence $\left\{V_{i(k)}\right\}_{i(k) \in \mathcal{I}}$ of non-empty sets $V_{i(k)} \subseteq \widehat{\mathbb{C}}, \widehat{\mathbb{C}}=\mathbb{C} \cup\{\infty\}$ is called the sequence of value regions of the BCF (1) with $b_{0}=0$ if

$$
a_{i(k)}\left(b_{i(k)}+\sum_{i_{k+1}=1}^{i_{k}} V_{i(k+1)}\right)^{-1} \subseteq V_{i(k)}, \quad i(k) \in \mathcal{I} .
$$

For the given sequence of value regions $\left\{V_{i(k)}\right\}_{i(k) \in \mathcal{I}}$ with $V_{i(k)} \subseteq \widehat{\mathbb{C}}$ sequence of regions $\left\{\Omega_{i(k)}\right\}_{i(k) \in \mathcal{I}}$, that are defined by the relations

$$
\Omega_{i(k)}:=\left\{(a, b) \in \mathbb{C}^{2}: a\left(b+\sum_{i_{k+1}=1}^{i_{k}} V_{i(k+1)}\right) \subseteq V_{i(k)}\right\}, \quad i(k) \in \mathcal{I},
$$

is called the sequence of elements regions of the $\mathrm{BCF}(1)$, where $b_{0}=0$, that are corresponding to the sequence of value regions $\left\{V_{i(k)}\right\}_{i(k) \in \mathcal{I}}$. The element region is a region from where the elements of the BCF are taken, that is $\left(a_{i(k)}, b_{i(k)}\right) \in \Omega_{i(k)}, i(k) \in \mathcal{I}$. If, in addition, the BCF (1) converges, then these regions are called convergence regions (see [18, p. 109]).

Theorem 4. Let for each $i(k), i(k) \in \mathcal{I}$,

$$
V_{i(k)}=\left\{z \in \mathbb{C}: \operatorname{Re}\left(z e^{-i \psi_{k}}\right) \geq-p_{i(k)}\right\}, \quad p_{i(k)}>0,
$$

and

$$
\Omega_{i(k)}=\left\{(a, b) \in \mathbb{C}^{2}:|a|-\operatorname{Re}\left(a e^{-i\left(\psi_{k}+\psi_{k+1}\right)}\right) \leq 2 p_{i(k)}\left(\operatorname{Re}\left(b e^{i \psi_{k+1}}\right)-p_{i(k)}^{*}\right)\right\},
$$

where $\pi / 2<\psi_{k}<\pi / 2, k=1,2, \ldots$, and

$$
p_{i(k)}^{*}=\sum_{i_{k+1}=1}^{i_{k}} p_{i(k+1)} \quad \text { for each } i(k) \in \mathcal{I} .
$$

Then for the BCF (1) with $b_{0}=0$ the $\left\{V_{i(k)}\right\}_{i(k) \in \mathcal{I}}$ and the $\left\{\Omega_{i(k)}\right\}_{i(k) \in \mathcal{I}}$ are sequences of value regions and elements regions respectively. 
The proof is carried out according to the scheme proposed in [7, p. 38] (see also [17]).

If the elements of the BCF (1) with $b_{0}=0$ satisfy the conditions $a_{i(k)} \in \mathbb{C}$ and $b_{i(k)} \in \mathbb{R}_{+}$for all $i(k) \in \mathcal{I}, \psi_{k}=0, k=1,2, \ldots$, then from Theorem 4 we immediately obtain the following result.

Corollary 1. Let for each $i(k) \in \mathcal{I}$

$$
V_{i(k)}=\left\{z \in \mathbb{C}: \operatorname{Re}(z) \geq-p_{i(k)}\right\}, \quad p_{i(k)}>0,
$$

and

$$
\Omega_{i(k)}=\left\{(a, b) \in \mathbb{C} \times \mathbb{R}_{+}:|a|-\operatorname{Re}(a) \leq 2 p_{i(k)}\left(b-p_{i(k)}^{*}\right)\right\},
$$

where $p_{i(k)}^{*}$ are defined in (8). Then for the BCF (1) with $b_{0}=0$ the $\left\{V_{i(k)}\right\}_{i(k) \in \mathcal{I}}$ and the $\left\{\Omega_{i(k)}\right\}_{i(k) \in \mathcal{I}}$ are sequences of value regions and elements regions respectively.

Let us establish parabolic regions of convergence for the BCF of the special form

$$
\left(b_{0}+D_{k=1}^{\infty} \sum_{i_{k}=1}^{i_{k-1}} \frac{a_{i(k)}}{b_{i(k)}}\right)^{-1}
$$

where $b_{0}, b_{i(k)}, a_{i(k)} \in \mathbb{C}, i(k) \in \mathcal{I}$.

Theorem 5. Let (9) be the BCF and for each $i(k) \in \mathcal{I}, b_{i(k)}$ is a fixed partial denominator chosen from

$$
\mathcal{B}_{i(k)}=\left\{z \in \mathbb{C}:|z| \geq p_{i(k)}^{*}\right\}
$$

where the $p_{i(k)}$ are some positive real numbers and $p_{i(k)}^{*}$ are defined in accordance with (8). And moreover $\operatorname{Re}\left(b_{0}\right)>\sum_{i_{1}=1}^{N} p_{i(1)}$. Suppose that the partial numerator $a_{i(k)}$ belongs to the parabolic region

$$
\mathcal{P}_{i(k)}=\left\{z \in \mathbb{C}:|z|-\operatorname{Re}\left(z e^{-i\left(\arg b_{i(k)}+\arg b_{i(k-1)}\right)}\right) \leq 2 p_{i(k)}(1-\varepsilon)\left(\left|b_{i(k)}\right|-p_{i(k)}^{*}\right)\right\},
$$

where $0<\varepsilon<1$ and $a_{i(k)} \neq 0$. Then

(A) there exist finite limits of the sequenses of even and odd approximants of the BCF (9);

(B) the $B C F(9)$ converges if the following series diverge

$$
\begin{gathered}
\sum_{p=1}^{\infty} \sqrt{\left|\frac{b_{m[p]} b_{m[p+1]}}{a_{m[p+1]}}\right|}, \quad m=\overline{1, N}, \\
\sum_{p=1}^{\infty} \sqrt{\mid \frac{b_{i(n), m[p]} b_{i(n), m[p+1]} \mid}{a_{i(n), m[p+1]} \mid}, \quad i(n)} \in \mathcal{I}^{(m+1)}, m=\overline{1, N-1} ;
\end{gathered}
$$

(C) all approximants of BCF (9) belong to the circle

$$
K=\left\{w \in \mathbb{C}:\left|w-\frac{1}{2}\left(\operatorname{Re}\left(b_{0}\right)-\sum_{i_{1}=1}^{N} p_{i(1)}\right)^{-1}\right| \leq \frac{1}{2}\left(\operatorname{Re}\left(b_{0}\right)-\sum_{i_{1}=1}^{N} p_{i(1)}\right)^{-1}\right\} .
$$


Proof. Using equivalent transformations [7, p. 29] (see also [18, p. 31]), where $\rho_{i(k)}=e^{-i \arg b_{i(k)}}$, $i(k) \in \mathcal{I}$, we reduce the BCF (9) to the following form

$$
\left(b_{0}+D_{k=1}^{\infty} \sum_{i_{k}=1}^{i_{k-1}} \frac{\tilde{a}_{i(k)}}{\tilde{b}_{i(k)}}\right)^{-1}
$$

where $\tilde{a}_{i(k)}=a_{i(k)} e^{-i\left(\arg b_{i(k)}+\arg b_{i(k-1)}\right)}, \tilde{b}_{i(k)}=\left|b_{i(k)}\right|, i(k) \in \mathcal{I}$. Taking into account the relation (10), it follows that the partial numerators of the BCF (12) belong to the parabolas

$$
P_{i(k)}=\left\{z \in \mathbb{C}:|z|-\operatorname{Re}(z) \leq 2 p_{i(k)}(1-\varepsilon)\left(\tilde{b}_{i(k)}-p_{i(k)}^{*}\right)\right\}, \quad i(k) \in \mathcal{I} .
$$

Using method of prooving of Theorem $3.22[7$, p. 111] let us prove that BCF (12) converges. We set $\tilde{a}_{i(k)}=\left|\tilde{a}_{i(k)}\right| e^{i \alpha_{i(k)}}, i(k) \in \mathcal{I}$. Let for each $i(k) \in \mathcal{I}$

$$
\tilde{a}_{i(k)}(z)=\left|\tilde{a}_{i(k)}\right| e^{i z \alpha_{i(k)}}, \quad z \in G_{\delta}
$$

where $G_{\delta}=\{z \in \mathbb{C}:|\operatorname{Im}(z)|<\delta,|\operatorname{Re}(z)|<1+\delta\}$, and the $\delta$ is an arbitrary real number such that $(1+\delta)^{2} e^{\pi \delta}(1-\varepsilon)<1$.

Now, let us prove that $\tilde{a}_{i(k)}(z) \in P_{i(k)}$, where $\varepsilon=0, i(k) \in \mathcal{I}$. We consider the BCF

$$
\left(b_{0}+D_{k=1}^{\infty} \sum_{i_{k}=1}^{i_{k-1}} \frac{\tilde{a}_{i(k)}(z)}{\tilde{b}_{i(k)}}\right)^{-1} .
$$

Using Corollary 1 we obtain that the value of the approximants of the BCF inverse to (13), belong to a half-plane

$$
V=\left\{z \in \mathbb{C}: \operatorname{Re}(z) \geq \operatorname{Re}\left(\tilde{b}_{0}\right)-\sum_{i_{1}=1}^{N} p_{i(1)}\right\} .
$$

Thus, the values of the approximants of the BCF (13) belong to the circle

$$
K=\left\{w \in \mathbb{C}:\left|w-\frac{1}{2}\left(\operatorname{Re}\left(\tilde{b}_{0}\right)-\sum_{i_{1}=1}^{N} p_{i(1)}\right)^{-1}\right| \leq \frac{1}{2}\left(\operatorname{Re}\left(\tilde{b}_{0}\right)-\sum_{i_{1}=1}^{N} p_{i(1)}\right)^{-1}\right\} .
$$

Let the $f_{n}(z)$ be the $n$th approximant of the BCF (13), $n=1,2, \ldots$ It is obvious that $f_{n}(z)$ is a holomorphic function in the domain $G_{\delta}$. For the sequence $\left\{f_{n}(z)\right\}$ the conditions of Theorem 2.17 [7, p. 66] hold, where, for example, $a=-1, b=-2$.

Let us take $\Delta=\{z \in \mathbb{C}: \operatorname{Re}(z)=0,|\operatorname{Im}(z)|<\delta\}$. Then for $z \in \Delta$ the BCF (13) has the form

$$
\left(b_{0}+D_{k=1}^{\infty} \sum_{i_{k}=1}^{i_{k-1}} \frac{a_{i(k)}^{\prime}}{\tilde{b}_{i(k)}}\right)^{-1}
$$

where $a_{i(k)}^{\prime}=\left|\tilde{a}_{i(k)}\right| e^{-\operatorname{Im}(z) \alpha_{i(k)}}, i(k) \in \mathcal{I}$.

From the divergence of the series (11), it follows the divergence of the series

$$
\sum_{p=1}^{\infty} \sqrt{\left|\frac{b_{m[p]} b_{m[p+1]}}{a_{m[p+1]}}\right|} e^{\alpha_{m[p]} \operatorname{Im}(z) / 2} \text { for each } m=\overline{1, N}
$$




$$
\sum_{p=1}^{\infty} \sqrt{\left|\frac{b_{i(n), m[p]} b_{i(n), m[p+1]}}{a_{i(n), m[p+1]}}\right|} e^{\alpha_{i(n), m[p]} \operatorname{Im}(z) / 2} \quad \text { for each } m=\overline{1, N-1}, i(n) \in \mathcal{I}^{(m+1)} .
$$

And this, according to Theorem 2, means that the BCF inverse to (14) is convergent, and hence the BCF (14) is convergent for $z \in \Delta$.

Thus, according to the Stieltjes-Vitali theorem [7, p. 62], the BCF (13) coincides on each compact subset of the domain $G_{\delta}$, in particular, on the compact set $\{1\}$, which is equivalent to convergence of the $\mathrm{BCF}(12)$, and hence of the $\mathrm{BCF}(9)$.

$\mathrm{BCF}$ of the special form with positive elements have the property of monotonicity of even and odd approximants. This fact could be proven analoguously as for general BCF [7, p. 29]. Therefore, we obtain that always there are finite limits of even and odd approximants of the BCF (14). And using the Stieltjes-Vitali theorem [7, p. 62], we make sure that the even and odd approximants of the BCF (9) have finite and equal limits.

Remark 1. The condition $a_{i(k)} \neq 0, i(k) \in \mathcal{I}$, can be omitted, since if there is a multiindex $i(k)$ such that $a_{i(k)}=0$, then the corresponding branch of the multidimensional fraction is terminated, and the series whose multiindexes of elements include $i(k)$ are not taken into account.

Theorem 5 is some kind of analogue of Theorem 3.22 [7, p. 111] reformulated for BCF of the special form.

Theorem 6. Let (9) be the BCF and for each $i(k) \in \mathcal{I}, b_{i(k)}$ is a fixed partial denominator chosen from

$$
\mathcal{B}_{i(k)}=\left\{z \in \mathbb{C}:|z| \geq p_{i(k)}^{*}\right\}
$$

where the $p_{i(k)}$ are some positive real numbers and $p_{i(k)}^{*}$ are defined in accordance with (8). And moreover $\operatorname{Re}\left(b_{0}\right)>\sum_{i_{1}=1}^{N} p_{i(1)} \cos \gamma$. Suppose that the partial numerator $a_{i(k)}$ belongs to the parabolic region

$$
\mathcal{P}_{i(k)}=\left\{z \in \mathbb{C}:|z|-\operatorname{Re}\left(z e^{-i\left(\arg b_{i(k)}+\arg b_{i(k-1)}+2 \gamma\right)}\right) \leq 2 p_{i(k)} \cos ^{2} \gamma(1-\varepsilon)\left(\left|b_{i(k)}\right|-p_{i(k)}^{*}\right)\right\},
$$

where $0<\varepsilon<1$ and $a_{i(k)} \neq 0$. Then

(A) there exist finite limits of the sequenses of even and odd approximants of the BCF (9);

(B) the BCF (9) converges if the series (11) diverge;

(C) all approximants of the BCF (9) belong to the circle

$$
K=\left\{w \in \mathbb{C}:\left|w-\frac{e^{-i \gamma}}{2}\left(\operatorname{Re}\left(b_{0}\right)-\sum_{i_{1}=1}^{N} p_{i(1)} \cos \gamma\right)^{-1}\right| \leq \frac{1}{2}\left(\operatorname{Re}\left(b_{0}\right)-\sum_{i_{1}=1}^{N} p_{i(1)} \cos \gamma\right)^{-1}\right\} .
$$

The proof of the theorem could be done according to the scheme of the proof of Theorem 3.23 [7, p. 114]. But before, by means of equivalent transformations, we reduce the BCF (9) to the equivalent BCF (12). The condition $a_{i}(k) \neq 0, i(k) \in \mathcal{I}$, can be omitted, taking into account Remark 1.

Putting $b_{i(k)}=1, i(k) \in \mathcal{I}, p_{i(2 s)}=\frac{1-d}{i_{2 s-1}}, p_{i(2 s+1)}=\frac{d}{i_{2 s}}, i(2 s), i(2 s+1) \in \mathcal{I}, s=1,2, \ldots$, $0<d<1$, we obtain a multidimensional analogue of Tron's theorem on twin parabolic domains of convergence [24]. 
Corollary 2. Let the elements of the $B C F$

$$
\left(b_{0}+D_{k=1}^{\infty} \sum_{i_{k}=1}^{i_{k-1}} \frac{a_{i(k)}}{1}\right)^{-1}
$$

belong to parabolic regions, that is $a_{i(k)} \in \mathcal{P}_{i(k)}, i(k) \in \mathcal{I}$, where

$$
\mathcal{P}_{i(k)}=\left\{z \in \mathbb{C}:|z|-\operatorname{Re}\left(z e^{-2 i \gamma}\right) \leq \frac{2 D_{k}^{2}(1-\varepsilon)}{i_{k-1}} \cos ^{2} \gamma\right\},
$$

where $D_{2 s}=(1-d)^{2}, D_{2 s-1}=d^{2}, 0<d<1, s=1,2, \ldots, 0<\varepsilon<1$. Then

(A) there exist finite limits of the sequenses of even and odd approximants of the BCF (15);

(B) the BCF (15) converges if the following series diverge

$$
\begin{gathered}
\sum_{p=1}^{\infty} \sqrt{\left|a_{m[p+1]}\right|^{-1}}, \quad m=\overline{1, N} \\
\sum_{p=1}^{\infty} \sqrt{\left|a_{i(n), m[p+1]}\right|^{-1}}, \quad i(n) \in \mathcal{I}^{(m+1)}, m=\overline{1, N-1}
\end{gathered}
$$

(C) all approximants of the BCF (15) belong to the circle

$$
K=\left\{w \in \mathbb{C}:\left|w-\frac{e^{-i \gamma}}{2\left(\operatorname{Re}\left(b_{0}\right)-d \cos \gamma\right)}\right| \leq \frac{1}{2\left(\operatorname{Re}\left(b_{0}\right)-d \cos \gamma\right)}\right\} .
$$

\section{Application}

The application of Corollary 2 will be illustrated by the study of the regions of convergence of multidimensional $S$-fractions with independent variables

$$
\left(1+D_{k=1}^{\infty} \sum_{i_{k}=1}^{i_{k-1}} \frac{a_{i(k)} z_{i_{k}}}{1}\right)^{-1}
$$

where $a_{i(k)}>0, i(k) \in \mathcal{I}, z_{i_{k}} \in \mathbb{C}, i_{k}=\overline{1, N}, \mathbf{z}=\left(z_{1}, z_{2}, \ldots, z_{N}\right) \in \mathbb{C}^{N}$.

The approximants $f_{n}(\mathbf{z})$ of these BCF are multidimensional rational functions. The following theorem investigates the convergence of these rational approximations. In this case, a multidimensional analogue of the Stieltjes-Vitali theorem [7, p. 62] is significantly used.

Theorem 7. Let the $\left\{f_{m}(\mathbf{z})\right\}$ be a sequence of holomorphic functions in the domain $D \subset \mathbb{C}^{n}$, uniformly bounded inside $D$.

If $f_{m}(\mathbf{z})$ converges at each point of the set $\Delta \subset D$, which is a $2 n$-dimensional neighborhood, an $n$-dimensional real or an n-dimensional imaginary neighborhood of the point $\mathbf{z}_{0} \in D$, then $f_{m}(\mathbf{z})$ converges uniformly on any compact set $K, K \subset D$, to a holomorphic function in $D$. 
Theorem 8. Let (16) be a multidimensional S-fraction with independent variables and the series

$$
\begin{gathered}
\sum_{p=1}^{\infty} a_{m[p]}^{-1 / 2}, \quad m=\overline{1, N} \\
\sum_{p=1}^{\infty} a_{i(n) m[p]}^{-1 / 2}, \quad i(n) \in \mathcal{I}^{(m+1)}, m=\overline{1, N-1},
\end{gathered}
$$

are divergent. Then

(A) if $\sup _{i(k) \in \mathcal{I}} a_{i(k)}=\infty$, then the BCF (16) converges in each point $\mathbf{z} \in G(\gamma)$, for each $|\gamma|<\pi / 2$,

$$
G(\gamma)=\underbrace{H(\gamma) \times H(\gamma) \times \cdots \times H(\gamma)}_{N} \subset \mathbb{C}^{N}, \quad H(\gamma)=\{w \in \mathbb{C}: \arg w=\gamma\},
$$

(B) if $\sup _{i(k) \in \mathcal{I}}\left(a_{i(k)} i_{k-1}\right)=A$, then the BCF (16) converges in the domain $P(\gamma)$, for each $|\gamma|<\pi / 2$

$$
\begin{gathered}
P(\gamma)=P_{1}(\gamma) \times P_{2}(\gamma) \times \cdots \times P_{N}(\gamma) \subset \mathbb{C}^{N} \\
P_{k}(\gamma)=\left\{z_{k} \in \mathbb{C}:\left|z_{k}\right|-\operatorname{Re}\left(z_{k} e^{(-2 i \gamma)}\right)<\frac{2 D_{k}}{A} \cos ^{2} \gamma\right\}, \quad k=\overline{1, N}
\end{gathered}
$$

where $D_{k}$ are defined in Corollary 2.

Proof. (A) Let $\gamma$ be an arbitrary real number such that $|\gamma|<\pi / 2$. Since the series (17) diverge, then for an arbitrary $\mathbf{z} \in G(\gamma)$ and an arbitrary $a_{i(k)}, i(k) \in \mathcal{I}$, the following series also diverge

$$
\begin{gathered}
\sum_{p=1}^{\infty}\left|a_{m[p]} z_{m}\right|^{-1 / 2} \text { for each } m=\overline{1, N} \\
\sum_{p=1}^{\infty}\left|a_{i(n) m[p]} z_{m}\right|^{-1 / 2} \text { for each } m=\overline{1, N-1}, i(n) \in \mathcal{I}^{(m+1)} .
\end{gathered}
$$

If $\mathbf{z} \in G(\gamma)$, then

$$
\left|a_{i(k)} z_{i_{k}}\right|-\operatorname{Re}\left(a_{i(k)} z_{i_{k}} e^{-2 i \gamma}\right)=0<\frac{2 D_{k}(1-\varepsilon)}{i_{k-1}} \cos ^{2} \gamma
$$

Thus, by Corollary 2 , the BCF (16) converges at each point $\mathbf{z} \in G(\gamma)$.

(B) Let $\left\{f_{n}(\mathbf{z})\right\}$ be the sequence of approximants of the BCF (16). Taking into account that $\sup _{i(k) \in \mathcal{I}}\left(a_{i(k)} i_{k-1}\right)=A$ we obtain for all $a_{i(k)}, i(k) \in \mathcal{I}$,

$$
\left|a_{i(k)} z_{i_{k}}\right|-\operatorname{Re}\left(a_{i(k)} z_{i_{k}} e^{-2 i \gamma}\right) \leq \frac{2 D_{k}}{i_{k-1}} \cos ^{2} \gamma
$$

It follows from Theorem 4 that

$$
\operatorname{Re}\left(1+D_{k=1}^{n} \sum_{i_{k}=1}^{i_{k-1}} \frac{a_{i(k)} z_{i_{k}}}{1}\right) \geq 1-d \cos \gamma
$$

Then the approximants of the BCF (16) are holomorphic functions in the domain $P(\gamma)$ and their values belong to the circle

$$
\mathcal{K}(\gamma)=\left\{w \in \mathbb{C}:\left|w-\frac{e^{-i \gamma}}{2(1-d \cos \gamma)}\right| \leq \frac{1}{2(1-d \cos \gamma)}\right\} .
$$


Therefore, the sequence $\left\{f_{n}(\mathbf{z})\right\}$ is bounded in $P(\gamma)$. Let

$$
\Delta=\{w \in \mathbb{C}: \arg w=2 \gamma, r<|w|<R\} .
$$

Obviously, $\Delta^{N} \subset P(\gamma)$. Then for an arbitrary $\mathbf{z} \in \Delta^{N}$, we have for all $a_{i(k)}, i(k) \in \mathcal{I}$,

$$
\left|a_{i(k)} z_{i_{k}}\right|-\operatorname{Re}\left(a_{i(k)} z_{i_{k}} e^{-2 i \gamma}\right)=0<\frac{2 D_{k}(1-\varepsilon)}{i_{k-1}} \cos ^{2} \gamma
$$

Since the series (18) are divergent, then according to Corollary 2 the BCF (16) converges if $\mathbf{z} \in \Delta^{N}$. By Theorem 7, the multidimensional S-fraction with independent variables (16) converges on each compact subsets of the domain $P(\gamma)$.

\section{Conclusions}

In the paper, we considered the problem of convergence of $\mathrm{BCF}$ of the special form. The problem of establishing new sufficient convergence criteria of BCF with complex elements in the case of numerical and functional BCF remains open. Concerning parabolic theorems, the question of the possibility of expanding these domains by omitting $\varepsilon$ is open, since the presence of $\varepsilon>0$ arises from the used method of proving. Therewith, the problem to establish truncation error bounds of a BCF, when its elements belong to parabolic domains, remains open.

\section{References}

[1] Antonova T.M. Multidimensional generalization of the theorem on parabolic domains of convergence of continued fractions. Mat. Met. Fiz.-Mekh. Polya 1999, 42 (4), 7-12. (in Ukrainian)

[2] Antonova T.M., Bodnar D.I. Convergence domains for branched continued fractions of the special form. Approx. Theor. and its Appl.: Pr. Inst. Matem. NAS Ukr. 2000, 31, 19-32. (in Ukrainian)

[3] Antonova T.M., Dmytryshyn R.I. Truncation error bounds for branched continued fraction $\sum_{i_{1}=1}^{N} \frac{a_{i(1)}}{1}+\sum_{i_{2}=1}^{i_{1}} \frac{a_{i(2)}}{1}+$ $\sum_{i_{3}=1}^{i_{2}} \frac{a_{i(3)}}{1}+$... Ukr. Math. J. 2020, 72 (7), 1018-1029. doi:10.1007/s11253-020-01841-7 (translation of Ukrain. Mat. Zh. 2020, 72 (7), 877-885. doi:10.37863/umzh.v72i7.2342 (in Ukrainian))

[4] Antonova T.M., Sus' O.M. On one criterion for the figured convergence of two-dimensional continued fractions with complex elements. J. Math. Sci. (N.Y.) 2010, 170 (5), 594-603. doi:10.1007/s10958-010-0104-x (translation of Mat. Metody Fiz.-Mekh. Polya 2009, 52 (2), 28-35. (in Ukrainian))

[5] Baran O.E. Some convergence regions of branched continued fractions of special form. Carpathian Math. Publ. 2013, 5 (1), 4-13. doi:10.15330/cmp.5.1.4-13 (in Ukrainian)

[6] Bilanyk I., Bodnar D. Convergence criterion for branched continued fractions of the special form with positive elements. Carpathian Math. Publ. 2017, 9 (1), 12-12. doi:10.15330/cmp.9.1.13-21

[7] Bodnar D.I. Branched continued fractions. Naukova Dumka, Kyiv, 1986. (in Russian)

[8] Bodnar D.I. On the convergence of branched continued fractions. J Math Sci. 1999,97 (1), 3862-3871. doi: 10.1007/BF02364926

[9] Bodnar O.S., Dmytryshyn R.I. On the convergence of multidimensional S-fractions with independent variables. Carpathian Math. Publ. 2018, 10 (1), 58-64. doi:10.15330/cmp.10.1.58-64

[10] Bodnar O., Dmytryshyn R., Sharyn S. On the convergence of multidimensional S-fractions with independent variables. Carpathian Math. Publ. 2020, 12 (2), 353-359. doi: 10.15330/cmp.12.2.353-359

[11] Bodnar D.I., Kuchminska Kh.Yo. Parabolic convergence region for two-dimensional continued fractions. Mat. Stud. 1995, 4, 29-36. (in Ukrainian) 
[12] Bodnarchuk P.I., Skorobohat'ko V.Ya. Branched Continued Fractions and Their Applications. Naukova Dumka, Kyiv, 1974. (in Ukrainian)

[13] Cuyt A., Verdonk B. A review of branched continued fraction theory for the construction of multivariate rational approximations. Appl. Numer. Math. 1988, 4 (2-4), 263-271. doi:10.1016/0168-9274(83)90006-5

[14] Dmytryshyn R.I., Antonova T.M. Truncation error bounds for branched continued fraction whose partial denominators are equal to unity. Mat. Stud. 2020, 54 (1), 3-14. doi:10.30970/ms.54.1.3-14

[15] Dmytryshyn R.I. Convergence of multidimensional A- and J-Fractions with independent variables. Comput. Methods Funct. Theory 2021. doi:10.1007/s40315-021-00377-6

[16] Dmytryshyn R.I. Multidimensional regular C-fraction with independent variables corresponding to formal multiple power series. Proc. Roy. Soc. Edinburgh Sect. A 2020, 150 (4), 1853-1870. doi:10.1017/prm.2019.2

[17] Jones W., Thron W. Convergence of continued fractions. Canad. J. Math. 1968, 20, 1037-1055. doi:10.4153/CJM1968-101-3.

[18] Jones W.B., Thron W.J. Continued fractions: analytic theory and applications. In: Encyclopedia of Mathematics and its Applications, 11 Addison-Wesley, Reading, MA, 1980.

[19] Khovanskii A.N. The applications of continued fractions and their generalizations to problems in approximation theory. Moscow, 1956. (in Russian)

[20] Kuchminska Kh.Yo. Two-dimensional continued fractions. Pidstryhach Institute for Applied Problems in Mechanics and Mathematics, NAS of Ukraine, Lviv, 2010. (in Ukrainian)

[21] Lorentzen L., Waadeland H. Continued fractions. Vol. 1. Convergence theory. Atlantis Press, Amsterdam, 2008.

[22] Murphy J.A., O’Donohoe M.R. A two-variable generalization of the Stieltjes-type continued fraction. J. Comput. Appl. Math. 1978, 4 (3), 181-190. doi:10.1016/0771-050X(78)90002-5

[23] Perron O. Die Lehre von den Kettenbrüchen. B. G. Teubner, Stuttgart, 1957. (in German)

[24] Thron W.J. Two families of twin convergence regions for continued fractions. Duke Math. J. 1943, 10 (4), $677-685$. doi:10.1215/S0012-7094-43-01063-4

[25] Siemaszko W. Branched continued fractions for double power series. J. Comput. Appl. Math. 1980, 6 (2), 121-125. doi:10.1016/0771-050X(80)90005-4

[26] Skorobogatko V.Ya. Theory of branched continued fractions and its applicatiot in computational mathematics. Nauka, Moscow, 1983. (in Russian)

[27] Wall H.S. Analytic theory of continued fractions. Van Nostrand, New York, 1948.

Received 16.10.2021

Боднар А.І., Біланик І.Б. Параболічні області збіжності гіллястих ланцюгових дробів спеціального вигляду // Карпатські матем. публ. - 2021. — Т.13, №3. - С. 619-630.

Використовуючи критерій збіжності гіллястих ланцюгових дробів спеціального вигляду 3 додатними елементами, встановлено ефективні достатні умови збіжності цих дробів. Аля вивчення параболічних областей збіжності використано техніку множин елементів та множин значень. Зокрема, у якості множин значень розглядаються півплощини. Встановлено багатовимірний аналог областей збіжності Трона для гіллястих ланцюгових дробів спеціального вигляду. Отримані результати дали змогу встановити умови збіжності багатовимірного $S$-дробу 3 нерівнозначними змінними.

Ключові слова і фрази: гіллястий ланщюговий дріб, збіжність, неперервний дріб, раціональне наближення. 